\title{
Stress and Environmental Health of Women in different Neighbourhoods of Lagos Metropolis
}

\author{
Nwokoro, I.I.C. ${ }^{1}$, Olayinka, D.N. ${ }^{2, *}$ and Okolie, C.J. ${ }^{2}$ \\ ${ }^{1}$ Department of Urban \& Regional Planning, Faculty of Environmental Sciences, University of Lagos, Nigeria \\ ${ }^{2}$ Department of Surveying \& Geoinformatics, Faculty of Engineering, University of Lagos, Nigeria \\ Corresponding Author: *nihinolayinka@yahoo.co.uk
}

\begin{abstract}
It has been established that women spend more time in the neighbourhood environment, and therefore, are more vulnerable to the observable poor conditions. The focus of this study is on neighbourhood environmental stressors that affect womens' health in Lagos metropolis. The factors considered include access to clean water, adequate sanitation, drainage conditions, ventilation and hygiene, type of energy for cooking and nutrition. These factors are exacerbated by poverty and differ across different neighbourhoods in Lagos metropolis. 1150 respondents (high50; medium - 328; and low - 772) consisting of randomly selected women, aged 18 years and above were selected from all the 17 Local Government Areas (LGAs) in metropolitan Lagos to achieve $100 \%$ representation. Focus Group Discussions were held with women from selected different neighbourhoods. A 5-point likert scale was used as a measure of self-reported stress and self-reported health, with higher numbers indicating a greater self-reported stress. From the different survey methods used, results show that women in the low income neighbourhoods are more vulnerable to environmental stressors, and so their health is mostly affected negatively. Women in the other income groups also experience some form of stress but at lower severity levels. Environmental stressors and severity of chronic illness are linked to stress. An improvement in the environmental conditions will reduce the amount of stress experienced by women of different income neighbourhoods.
\end{abstract}

Keywords: Stress, Environmental Stressors, Metropolitan Lagos, Neighbourhoods, Health

\subsection{Introduction}

Women are major players in health care service provision through their roles as household managers and carers. In so doing, they spend longer hours in their household environment. All societies are divided along what we can call the fault line of gender (Moore, 1988; Papenek, 1990). This means that women and men are defined as different types of beings, each with their own opportunities, roles and responsibilities. However, WHO (1994) notes that these circumstances cause women to cope with the pressures of modernisation, which often requires them to assume additional duties and responsibilities, plus the burdens of their traditional roles. Thus women tend to have less time and energy. The social role of women may make them more vulnerable to certain hazards or exposures. Examples are the stress of womens' multiple roles as income providers, home managers, and reproducers; or poor nutritional status which can increase susceptibility to environmental chemicals such as lead and cadmium, and exposure to harmful emissions from smoke while cooking. This was corroborated in the theory of work-family-conflict by Greenhaus and Beutell (1985). The above indices are the major reasons for exploring the interplay between the neighbourhood environment, stress and health of women.

Pearlin and Schooler (1978) reported that the concept of stress not only refers to major life events but also encompasses ongoing minor events like electricity failure, maids not turning up, unexpected guests and childrens' misbehaviour. Similarly, stress can be viewed as a physiological demand placed on the body when one must adapt, cope or adjust (Nevid and Rathus, 2007). Different types of stress include psychological, physical, chemical, environmental, long term and short term stress (Kelly et 
al., 1997). Similarly, Djuric et al. (2008) have also noted that chronic stressors (a long term form of stress) associated with health disparities include perceived discrimination, neighbourhood stress, daily stress, family stress, acculturative stress, environmental stress and maternal stress. However, this study focuses on the environmental stress particularly how neighbourhood environmental factors interact with a woman's individual genetic susceptibilities to affect her health over her lifetime. The factors considered include access to clean water, adequate sanitation, drainage conditions, ventilation and hygiene, type of energy for cooking and nutrition.

Evidence strongly suggests that the neighbourhood in which people live influences their health, either in addition to or in interaction with individual level characteristics (Vidanaarachchi et al., 2006). There are environmental health challenges in Lagos which include low access to potable water, poor sanitation methods, inadequate drainage provision and poor housing conditions particularly in low income communities. These factors which are exacerbated by poverty differ across different neighbourhoods in Lagos and women and children are more vulnerable to these conditions. Similarly, in the study of prevalence of different neighbourhood environmental stressors and associations between the stressors and self-rated health, nuisance from neighbours and drug users, shortage of water and having poor water/sewage drainage system were associated with self-rated mental health among the women (Perera et al., 2009).

This aspect of womens' health is under-researched in the developing countries like Nigeria. It is therefore important to understand stress, especially as it relates to gender and health. This study investigates how these environmental stress conditions affect the health of women in different income residential neighbourhoods of metropolitan Lagos. It specifically attempts to investigate which environmental stressors are mostly experienced by women, what the most prevalent reported environmentally related diseases are, and how these affect the health status of women in different neighbourhoods of Lagos.

Although there are many different concepts of stress in the respective fields of medicine, psychology, and sociology, it is generally understood that stress is aversive in some sense. Psychologists often favour the stress concept proposed by Lazarus (1993) which states that stress is a condition or feeling experienced when a person perceives that demands exceed the personal and social resources the individual is able to mobilize. Kelly et al. (1997) identified the principal components of stress as stressors and the stress response. Stressors can be broadly defined as those events or situations that perturb a person's psychological and/or physical homeostasis. The authors believe that the division of labour by gender results in differential exposure and vulnerability to stress among women and men; women are in poorer health because their lives are more stressful than that of men and they are more vulnerable to the health consequences of life stressors because of their relative lack of material, personal, and social resources.

Some researchers argue that the health effects of stress may be experienced and embodied by women and men in different ways (Umberson et al., 1996). An investigation done by Macintyre et al. (1996) using data from a British regional, longitudinal study found female excess in ill health for depression, high blood pressure, varicose veins, malaise symptoms (such as worrying, nervousness, difficulty in concentrating, tiredness, and sleep problems), as well as selected physical symptoms (such as headaches, fainting, or dizziness). However, environmental stress which is the major focus of this study is constructed from some items that indicate problems with residential neighbourhoods and friends.

"The living environment plays a vital role in determining health" (Stafford et al., 2006; Wedan et al., 2008). As also reported by Rao et al. (2007) and Wedan et al. (2008), individuals living in poor neighbourhood environments tend to have higher morbidity and mortality rates compared to those living in environmentally sound neighbourhoods. Accordingly, adverse neighbourhood factors have been shown to be positively associated with coronary heart disease (CHD) (Diez-Roux et al., 1997; Sundquist et al., 2006). The authors further observed that "neighborhood economic deprivation may compromise health-promoting resources" (Diez-Roux et al., 2001). For example, poor and minority neighbourhoods tend to have fewer grocery stores with healthy foods (Morland et al., 2002) and fewer pharmacies with needed medications (Morrison et al., 2000). Finally, poor nutrition can increase susceptibility to environmental pollutants by compromising immune functions (Rios et al., 1993). Figure 1 illustrates a stress-exposure disease framework for environmental health disparities. 
Also, the study carried out by Hazra et al. (2005) titled "effects of household environment on womens' health in Northeast India" clearly illustrates the morbidity situation mainly attributable to household air pollution. It tells how the factors related to the household environment influence the prevalence of diseases and thus the health of women. However, most researches in Nigeria have focused mainly on occupational stress and health. For example, Mojoyinola (2008) investigated the effects of job stress on health of nurses in Ibadan and found that the highly stressed nurses exhibited personal and work behavioural problems. Similarly, Oluwole et al. (2012) examined the relationships between stress, social support and work/family conflict on the mental health of Nigerian women. The study revealed that there was significant difference between young and old women in the level of stress experienced; as well as between junior and senior staff in the social support experienced between single and married women. This study, therefore, intends to further explore the relationship between environmental stressors and health of women across different neighbourhoods of Lagos metropolis.

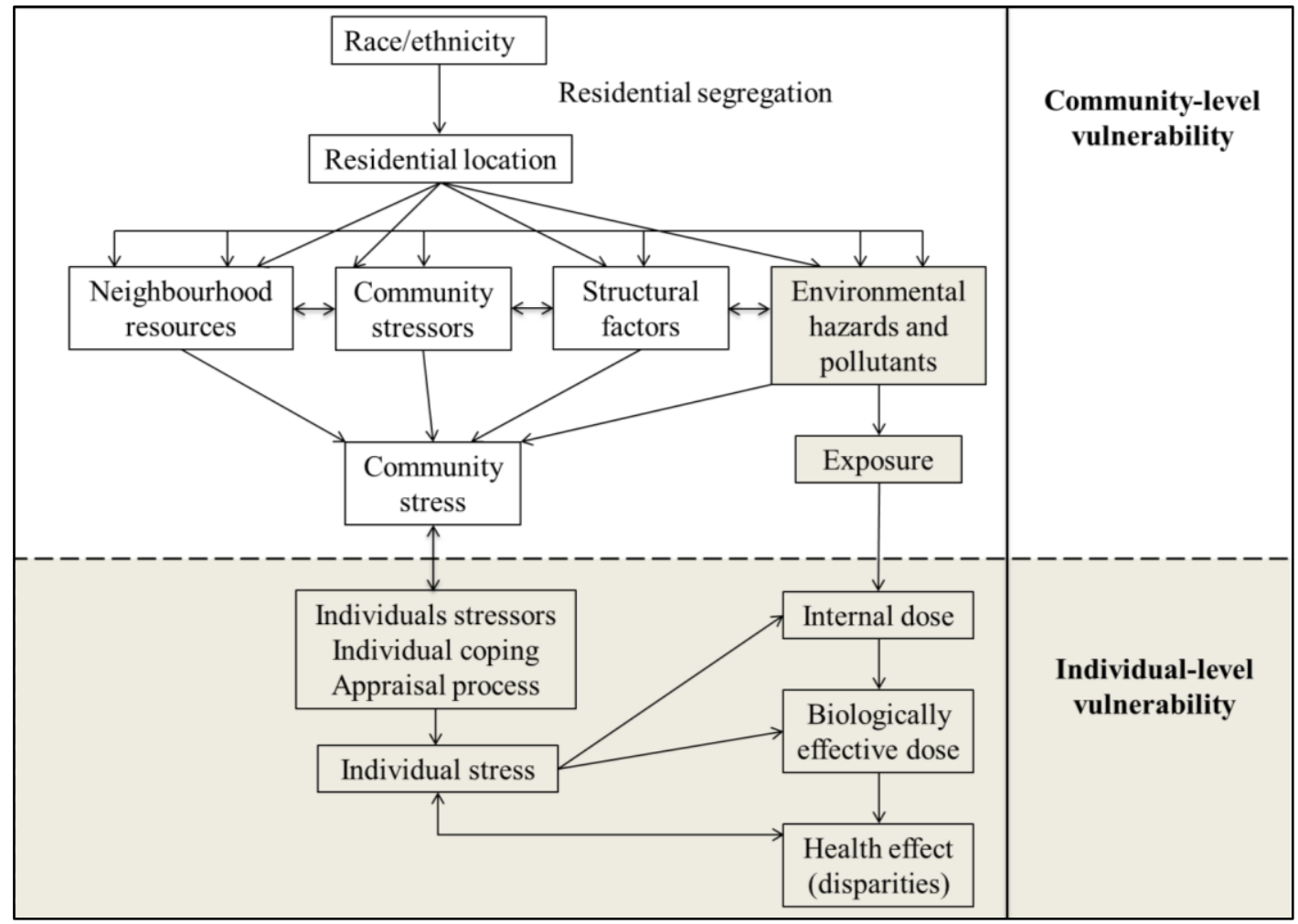

Figure 1: Stress-exposure disease framework for environmental health disparities Source: Modified from NIEHS (2004)

\subsection{Methodology}

\subsection{Study area}

The seventeen Local Government Areas (LGAs) in Lagos metropolis were selected in order to achieve $100 \%$ representation. Collectively, these seventeen LGAs had a total population of 4,129,697 females at the 2006 national census (National Bureau of Statistics, 2012). This figure represents 94\% of the total female population in the state recorded by the census. For the questionnaire distribution, one LGA each from the most predominant income neighbourhoods in the metropolis was selected. The three LGAs selected include Eti-osa representing the high income area, Lagos Mainland for the medium income area and Alimosho for the low income area. These three LGAs have a combined female population of 934,886 persons. A map of Lagos State showing the metropolis is presented in Figure 2. Lagos State was the former capital of Nigeria and is the country's centre of commerce. The state has a very diverse and fast-growing population, resulting from heavy and ongoing migration to its cities from all parts of Nigeria as well as neighbouring countries. The metropolitan area of the state lies between Latitudes $6^{\circ} 20^{\prime} 00^{\prime \prime}-6^{\circ} 42^{\prime} 10^{\prime \prime} \mathrm{N}$ and Longitudes $3^{\circ} 02^{\prime} 30^{\prime \prime}-3^{\circ} 42^{\prime} 40^{\prime \prime} \mathrm{E}$. It comprises the following LGAs - Agege, Ajeromi/Ifelodun, Alimosho, Amuwo Odofin, Apapa, Eti-Osa, Ifako/Ijaye, Ikeja, Ikorodu, Kosofe, Lagos Island, Lagos Mainland, Mushin, Ojo, Oshodi/Isolo, Shomolu, and Surulere. 


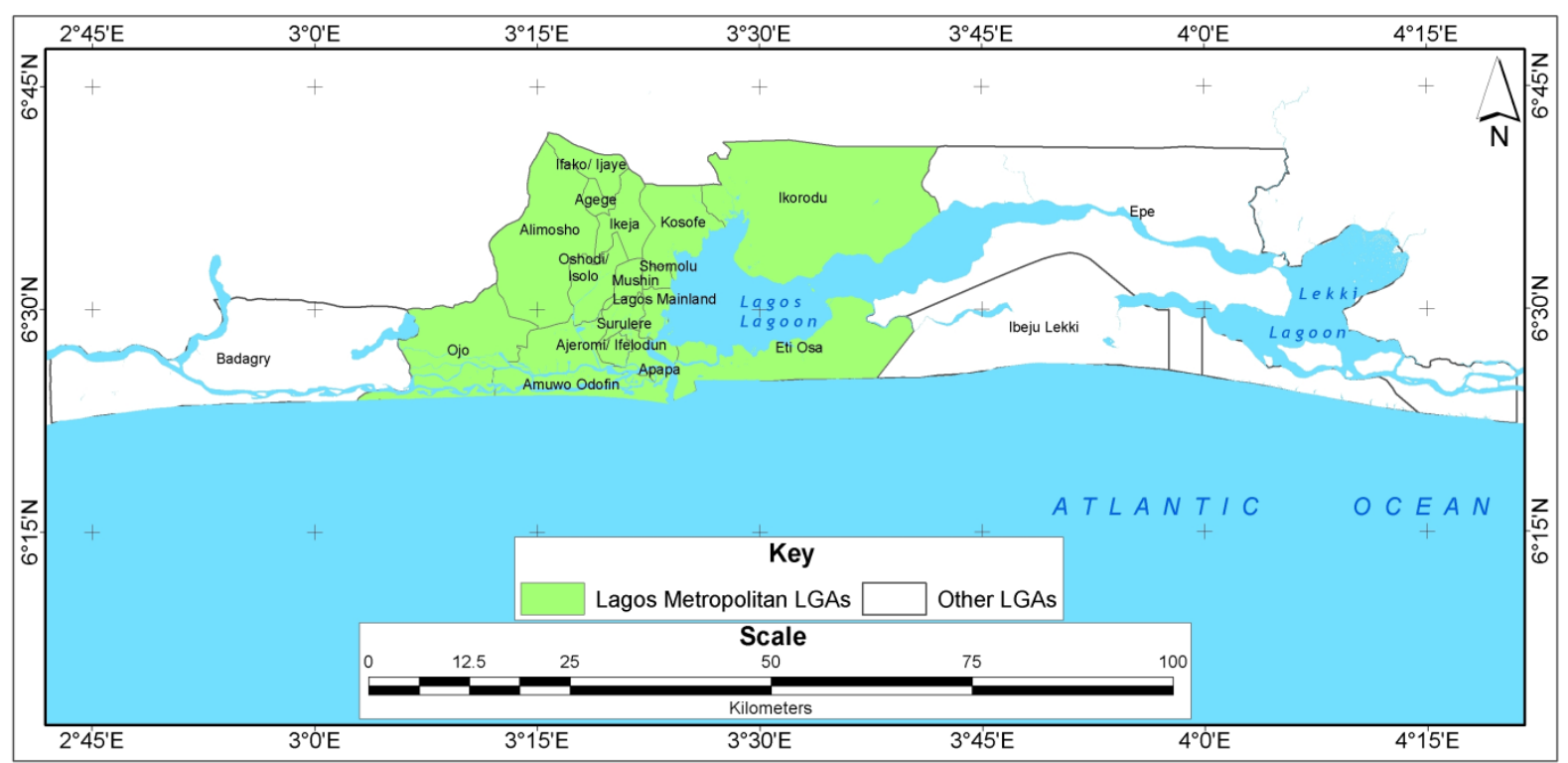

Figure 2: Map of Lagos State showing the LGAs in the metropolis

\subsection{Procedure}

A multi stage sampling method was used to arrive at a selection of 1150 respondents made up of high (50), medium (328) and low (772) income neighbourhoods comprising seventy-two wards within the three selected LGAs. Questionnaires were administered to a random selection of women aged 18 years and above. Focus Group Discussions were also held with women from different neighbourhoods. A 5-point likert scale was used as a measure of self-reported stress and self-reported health, with higher numbers indicating a greater self-reported stress. To facilitate the interpretability of interactive effects, the Cronbach's alpha, a measure of internal reliability, bound by 0 and 1 , with measures closer to 1 representing strong reliability for the items in the research instrument was adopted. Data analysis was by descriptive statistics, chi-square tests, and mean item scores (for the likert scale).

For the Focus Group Discussion, one LGA each from the most predominant income neighbourhood was selected. The three LGAs selected include Eti-osa representing the high income area, Lagos Mainland for the medium income area and Alimosho for the low income area. Accordingly, two groups were chosen from the high income area, three groups from the medium income area and four groups from the low income area. Each group consisted of ten women of age 18 years and above drawn from different wards, educational, social and professional backgrounds. Also each group was met four times to ascertain consistency of comments. The choice of the number of groups from each LGA was informed by findings in the literature (e.g. Nwokoro and Agbola, 2011) which showed that women from the low income area are more vulnerable to environmental health problems.

\subsection{Results and Discussion}

The major issues discussed here include the socio-economic characteristics of the women, the environmental stressors and their relationship with the health of women. Results of the likert scale were also discussed. Finally, results of the Focus Group Discussions are presented to corroborate the research questionnaire data.

\subsection{Socio-economic characteristics of the women}

The major factors considered here are the womens' monthly income and their educational status. Table 1 shows all the socio-economic variables considered in this study. While over $40 \%$ of women in the low income neighbourhood earn less than $\$ 15,000$ (\$100) per month, about $75 \%$ of the women in the high income areas earn over $\$ 45,000$ (\$300) per month. 
Table 1: Selected Socio-economic characteristics of women

\begin{tabular}{lccc}
\hline $\begin{array}{l}\text { Socio-economic } \\
\text { Characteristics }\end{array}$ & $\begin{array}{c}\text { Low Income } \\
\text { (LI) }\end{array}$ & $\begin{array}{c}\text { Medium Income } \\
\text { (MI) }\end{array}$ & $\begin{array}{c}\text { High Income } \\
\text { (HI) }\end{array}$ \\
\hline Income per Month (Naira) & & & \\
\hline$<15,000$ & 40.1 & 18.4 & 5.0 \\
$15,000-30,000$ & 27.2 & 14.4 & 7.5 \\
$30,001-45,000$ & 8.6 & 18.4 & 15.0 \\
$>45,000$ & 24.1 & 48.9 & 72.5 \\
Total & 100 & 100 & 100 \\
\hline Level of Education & & & \\
\hline No Formal Education & 7.8 & 4.9 & 2.5 \\
Primary School & 11.6 & 10.8 & 5.0 \\
Secondary School & 38.7 & 25.9 & 17.5 \\
Tertiary Education & 36.1 & 47.5 & 62.5 \\
Others & 5.9 & 10.8 & 12.5 \\
Total & 100 & 100 & 100 \\
\hline No. of respondents (N) & 732 & 305 & 40 \\
\hline Source: Questionnaire survey (2012) & & &
\end{tabular}

There is also a significant difference between the earning capacities of income of the women across the income groups. Results of the chi-square tests $\left(\pi^{2}=79.777 ; \mathrm{P}=0.05\right)$ corroborates the relationship between the income earned and residential neighbourhoods of women. Women in the high income group attained the highest levels of education more than those in the low income group. There is a significant difference in the levels of educational attainment of the various income groups as shown in Table 1 and results of the chi-square tests $\left(\pi^{2}=49.238 ; \mathrm{P}=0.05\right)$. This means that the different income groups attained different levels of education. With the above results, it can be concluded that the socio-economic group of women is a function of income and educational status.

\subsection{Environmental stressors and health of the women}

Having identified the environmental stressors affecting the women in different neighbourhoods, an investigation of the relationship between these stressors and the health of the women was done using the most reported environmentally related diseases as shown in Table 2 . The results revealed that the low income group experienced all the diseases most, followed by the medium income group and the least by the high income group. All the diseases examined are also stress inducing. It can be concluded that the environmental stressors are associated with the health of the women but at different degrees.

Table 2: Most frequently reported experienced environmental diseases in women (occurring more than 4 times in 1 year)

\begin{tabular}{lccc}
\hline $\begin{array}{l}\text { Types of Environmental } \\
\text { Diseases (\%) }\end{array}$ & Low Income (LI) & Medium Income (MI) & High Income (HI) \\
\hline Malaria & 44.5 & 33.8 & 17.5 \\
Diarrhoea & 70.5 & 54.4 & 35.0 \\
Sleeplessness & 37.4 & 51.8 & 87.5 \\
Fatigue & 25.1 & 36.4 & 85.0 \\
Respiratory Infection & 77.5 & 49.8 & 42.5 \\
Total & 100 & 100 & 100 \\
\hline
\end{tabular}

Note : Multiple responses possible

Source: Questionnaire survey (2012)

Figure 3 shows the distribution of these most frequently reported experienced environmental diseases presented graphically on a map of the Focus LGAs. To further explore the relationship between stress, environmental factors and health of the women in Lagos, a 5-point likert scale was used to elicit answers from questions on the most stressful environmental stressor in the past one year on one hand, and the effect of these environmental stressors on their health on the other. Responses ranged from 1 (not stressful) to 5 (most stressful) across the different neighbourhoods. This was further subjected to analysis using the mean item score, which led to the ranking of the environmental factors according to 
the most stressful (items with the highest score) to the least stressful. These are indicated as selfreported stress and self-reported health in Tables 3 and 4 respectively.

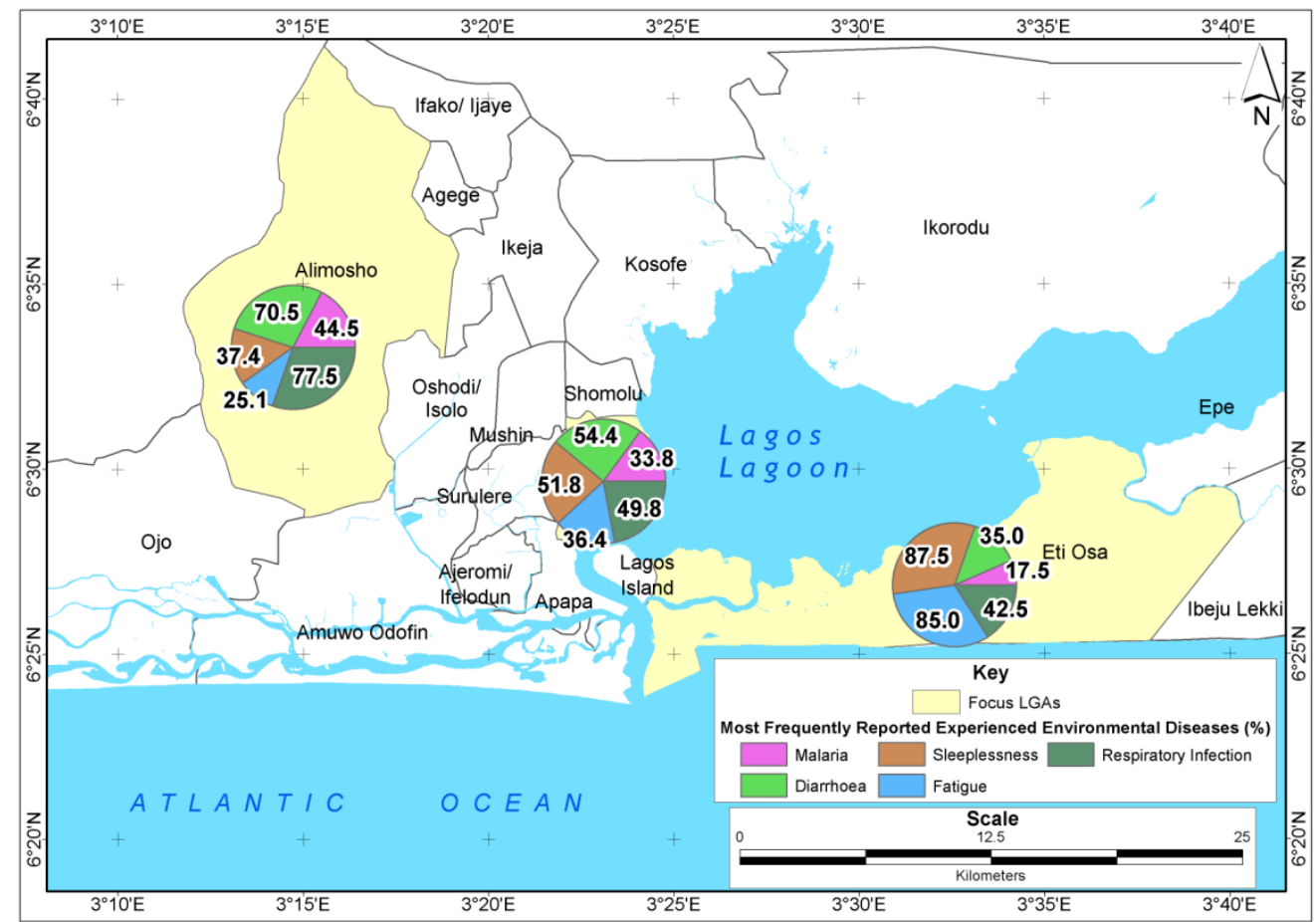

Figure 3: Distribution of the most frequently reported experienced environmental diseases shown graphically on a map of the Focus LGAs

These results are corroborated by findings from earlier studies. For example, Nwokoro and Agbola (2011) while examining the environment and health inequalities of women in different neighbourhoods of metropolitan Lagos reported that the women across all income levels experienced some diseases associated with poor environmental conditions. Their findings also showed that the health status of women in the high and medium income communities were higher than that of the women in the low- income communities (slums).

Table 3: Self-reported stress (most stressful environmental stressor in the past one year)

\begin{tabular}{|c|c|c|}
\hline Environmental factors & Mean Item Score & Ranking \\
\hline \multicolumn{3}{|l|}{ Low Income } \\
\hline Water quality and supply & 0.881 & 1 \\
\hline Ventilation and hygiene & 0.79 & 2 \\
\hline Cooking source of energy & 0.755 & 3 \\
\hline Drainage facilities & 0.696 & 4 \\
\hline Nutrition (food) & 0.648 & 5 \\
\hline \multicolumn{3}{|l|}{ Medium Income } \\
\hline Ventilation and hygiene & 0.712 & 1 \\
\hline Cooking source of energy & 0.704 & 2 \\
\hline Water quality and supply & 0.685 & 3 \\
\hline Drainage facilities & 0.657 & 4 \\
\hline Nutrition (food) & 0.525 & 5 \\
\hline \multicolumn{3}{|l|}{ High Income } \\
\hline Ventilation and hygiene & 0.575 & 1 \\
\hline Cooking source of energy & 0.57 & 2 \\
\hline Nutrition (food) & 0.55 & 3 \\
\hline Water quality and supply & 0.535 & 4 \\
\hline Drainage facilities & 0.415 & 5 \\
\hline
\end{tabular}

Source: Questionnaire survey (2012) 
Results from Table 3 show that the most significant environmental stressor reported by the low income group is poor water quality and supply with a mean item score of 0.881 . Other significant environmental stressors reported by this group of women are poor ventilation and hygiene and sources of energy for cooking which ranked second and third respectively. The practical implication of this result is that current conditions of environmental factors in the low income neighbourhood are very poor and there is an urgent need for improvement. On the other hand, the middle income group reported poor ventilation and hygiene and poor sources of energy for cooking as the most stressful environmental factors. These were significant with mean item scores of 0.718 and 0.704 , and ranked first and second respectively, closely followed by poor sources of water. Results of this group of women appears better in terms of stress level, but the environmental conditions still need to be improved on. The results of the high income group of women do not show any of the environmental factors having a significant stress impact. However, ventilation and hygiene ranked highest with a mean item score of 0.575. Again, this corroborates the results of the questionnaire survey indicating that this group of women have access to good sources of water, drainage facilities and, sources of energy for cooking.

Table 4: Self-reported health (how much have these environmental stressors affected your health in the past one year)

\begin{tabular}{lcc} 
Environmental factors & Mean Item score & Ranking \\
\hline Low Income & & 1 \\
\hline Water quality and supply & 0.861 & 2 \\
Nutrition (food) & 0.811 & 3 \\
Ventilation and hygiene & 0.809 & 4 \\
Cooking source of energy & 0.76 & 5 \\
Drainage facilities & 0.681 & \\
\hline Medium Income & & 1 \\
\hline Ventilation and hygiene & 0.718 & 2 \\
Cooking source of energy & 0.704 & 3 \\
Water quality and supply & 0.685 & 4 \\
Drainage facilities & 0.633 & 5 \\
Nutrition (food) & 0.622 & 1 \\
\hline High Income & & 2 \\
\hline Drainage facilities & 0.685 & 3 \\
Water quality and supply & 0.665 & 4 \\
Nutrition (food) & 0.63 & 5 \\
Ventilation and hygiene & 0.51 & \\
Cooking source of energy & 0.505 & \\
\hline
\end{tabular}

Source: Questionnaire survey (2012)

Similarly, results of ranking of the self-reported health by different groups of women are shown in Table 4. For the low income group, poor sources of water $(0.861)$, poor nutrition $(0.811)$ and poor ventilation and hygiene (0.809) were reported as the most significant environmental factors that affected their health in the past one year. Poor sources of energy for cooking was also reported as having some effect on their health. These also agree with previous results which identified these factors as the most reported environmental stressors in the past one year for this group of women. However, the most reported stressful environmental factors to the health of the women in the medium income group are poor ventilation and hygiene (0.718) and poor sources of energy for cooking (0.704), followed by poor sources of water. These were the same factors reported by the women in this group as causing them the highest level of stress. Again, the environmental stressors as reported by the high income group appear to have the least effect on the health of this group. Although poor drainage facilities and poor sources of water ranked high on the scale, their mean item scores are not as high as those of the low and medium income groups. It can be concluded that women in all income groups experience stress associated with environmental factors which also affect their health but these occur at varying degrees. 
The Focus Group Discussions identified stress as a major cause of ill health among women across different income neighbourhoods although reported at various levels. Women from all the three groups said that stress, in addition to poor environmental conditions, affects their health. This is in consonance with the findings by Nwokoro and Okusipe (2002) that deteriorating environmental conditions (poor access to safe water, waste management disposal methods, affordable healthcare) are the major contributory factors to poor health and quality of life in Lagos metropolis. The women further said that although the condition of the environment can affect their health, there are other associated factors of which stress is the most prominent. As already discussed in the literature review, stress can take on many different forms, and can contribute to symptoms of illness. Common symptoms of stress include headache, sleep disorders, difficulty in concentration, short temper, upset stomach, job dissatisfaction, low morale, depression, and anxiety. In the focus groups, women noted that certain socio-economic factors may contribute to the symptoms of stress. For example, individuals with less education may be at a higher risk of stress. The unemployed and those with little access to healthcare are also more likely to experience stress. When one's livelihood is at stake, the simple act of survival can be stressful. Accordingly, the Focus Group Discussion participants explained that their daily activities had no room for rest. In spite of their busy schedule as workers and children minders, they are still expected to take care of their husbands. The interesting aspect of this is that stress cuts across all the income groups, although reported at different levels. As the women agreed, this is caused by their changing roles in society.

This is well captured by participant 7, a middle aged woman in the low income group who stated that:

"Stress is a major cause of ill-health for me. In the morning before 5am, I wake up, bath the children and prepare them for school, cook for the family, and then go to market where I hawk. I do not have a house help to assist in all these chores and my husband does not care to help. I get home late to continue caring for the family."

The major difference between the three Focus Group Discussion reports of women from different income groups is the ability and resources to manage the stress. The professional women in the high income group are able to afford the services of house helps which to an extent reduces their stress and consequently lessens the impact on their health. Here lies the inequality in their health status.

\subsection{Conclusions}

This study has explored the socio-economic as well as the environmental conditions of women in different income neighbourhoods of Lagos metropolis. It further $\mathrm{x}$-rayed the relationship between stress, environmental factors and the health of these women. From the different survey methods used, results show that women in the low income neighbourhoods are more vulnerable to environmental stressors, and as such their health is mostly affected negatively. Women in the other income groups also experience some form of stress but at lower severity levels. Environmental stressors and severity of chronic illness are linked to stress. An improvement in the environmental conditions will reduce the amount of stress experienced by women of different income neighbourhoods.

\section{Acknowledgements}

The authors are grateful to the African Population Health Research Centre (APHRC), Kenya for their support in hosting the lead researcher on this study.

\section{References}

Diez-Roux, F.J., Nieto, C., Muntaner, H.A., Tyroler, G.W., Comstock, E., Shahar, L.S., Cooper, R.L., and Watson, S.M. (1997). Neighbourhood environments and coronary heart disease: a multilevel analysis. Am J Epidemiol, 146(1):48-63.

Diez-Roux, A.V., Merkin, S.S., Arnett, D., Chambless, L., Massing, M., and Nieto, F.J. (2001). Neighbourhood of residence and incidence of coronary heart disease. N Engl J Med, 345:99-106. 
Djuric, Z., Bird, C.E., Furumoto-Dawson, A., Rauscher, G.H., Ruffin, M.T., Raymond, I.V., Stowe, P., Tucker, K.L., and Masi, C.M. (2008). Biomarkers of psychological stress in health disparities research. The Open Biomarkers Journal, 1:7-19.

Greenhaus, J., and Beutell, N. (1985). Sources of conflict between work and family roles. Academy of Management Revie, 10: 76-88.

Hazra, A., Datta, S., and Guha, M. (2005). Effect of household environment on women's health in Northeast India. International Institute for Population Sciences. Mumbai.

Kelly, S., Hertzman, C., and Daniels, M. (1997). Searching for biological pathway between stress and health. Annual Review of Public Health, 18:437-62

Lazarus, R.S. (1993). Why we should think of stress as a subset of emotion. In: Leo Golderberger and Shlomo Breznitz (Eds). The Handbook of Stress The Free Press, New York, USA.

Macintyre, S., Hunt, K., and Sweeting, H. (1996). Gender differences in health: are things really as simple as they seem? Social Science \& Medicine, 42: 617-624.

Mojoyinola, J.K. (2008). Effects of job stress on health, personal and work behaviour of nurses in public hospitals in Ibadan Metropolis, Nigeria. Kamla-Raj (Ethno-Med), 2(2):143-148.

Moore, H. (1988). Feminism and Anthropology. Polity Press, Oxford.

Morland, K, Wing, S., and Diez-Roux, A. (2002). Neighborhood characteristics associated with the location of food stores and food service places. Am J Prev Med, 22:23-29.

Morrison, R.S., Wallenstein, S., Natale, D.K., Senzel, R.S., and Huang, L.L. (2000). We don't carry that - failure of pharmacies in predominantly non-white neighbourhoods to stock opioid analgesics. $N$ Engl J Med, 3426:1023-1026.

National Bureau of Statistics (2012). Annual abstract of statistics, 2012.

Nwokoro, I.I.C., and Agbola, B.S. (2001). Environment and health inequalities of women in different neighbourhoods of Metropolitan Lagos, Nigeria. In Maantay, J., \& McLafferty, S. (Eds) Geospatial Analysis of Environmental Health - Geo technologies and the Environment, USA, Springe, pps 283-302, USA.

Nwokoro, I.I.C., and Okusipe, M.D. (2002). Urban health and urban infrastructure: A spatial analysis of low-income communities in Lagos metropolis. In Amole, D., Ajayi, A., \& Okewole, A (Eds): The City in Nigeria. Faculty of Environmental Management, Obafemi Awolowo University, Ile-Ife, Nigeria, pps 378-383.

Nevid, J.S., and Rathus, S.A. (2007). Your Health. In: Mason, O. H: Thomson Custom Solutions.

Oluwole, DA., Hammed, A.T., and Awaebe, J.I. (2012). Patterns of stress, social support, and mental health among Nigerian women. Advancing Women in Leadership Journal, March 2012, Kindle Edition.

Papenek, H. (1990). To each less than she needs, from each more than she can do; allocations, entitlements and value. Tinker, I. [Ed] Persistent Inequalities; Women and World Development. Oxford University Press, Oxford.

Pearlin, L., and Schooler, C. (1978). The structure of coping. J. Hlth. Soc.Behav., 19: 2- 21.

Perera, B., Qstbye, T., and Jayawardana, C. (2009). Neighborhood environment and self-rated health among adults in southern Sri Lanka .Int. J. Environ. Res. Public Health, 6: 2102-2112.

Rao, M., Prasad, S., Adshead, F., and Tissera, H. (2007). The built environment and health. BMJ, 370:1111-1113.

Rios, R., Poje, G.V., and Detels, R. (1993). Susceptibility to environmental pollutants among minorities. Toxicol Ind Health, 9:797-820. 
Stafford, M., and McCarthy, M. (2006). Neighborhoods, housing and health. In: Marmot M, Wilkinson RG, (Eds). Social determinants of health. 2nd ed. Oxford University Press; Oxford, UK. 297-317.

Sundquist, K., Theobald, H., Yang, M., Li, X., Johansson, S.E., and Sundquist, J. (2006). Neighborhood violent crime and unemployment increase the risk of coronary heart disease: a multilevel study in an urban setting. Soc Sci Med, 62(8):2061-2071.

Umberson, D., Chen, M.D., House, J.S., Hopkins, K., and Slaten, E. (1996). Gender differences in relationships and psychological well-being. American Sociological Review, 61: 837-857.

Vidanaarachchi, C.K., Yuen, S.T., and Pilapitiya, S. (2006). Municipal solid waste management in the southern province of Sri Lanka; problems, issues and challenges. Waste Manage, 26:920-930.

Wedan, M.M., Carpiano, R.M., and Robert, S.A. (2008). Subjective and objective neighbourhood characteristics and adults health. Soc. Sci. Med, 66:1256-1270.

World Health Organization (1994). Assessment of fracture risk and its application to screening for post-menopausal women. Technical Paper Series No. 843. WHO Scientific Study Group, Geneva. 